Document downloaded from:

http://hdl.handle.net/10251/177487

This paper must be cited as:

Ureña-Gisbert, M.; García-Cortijo, S.; Gasulla Mestre, I. (2020). Space-Division Multiplexing Fibers for Microwave Signal Processing. IEEE. 1-2.

https://doi.org/10.1364/CLEO_SI.2020.STu3R.1

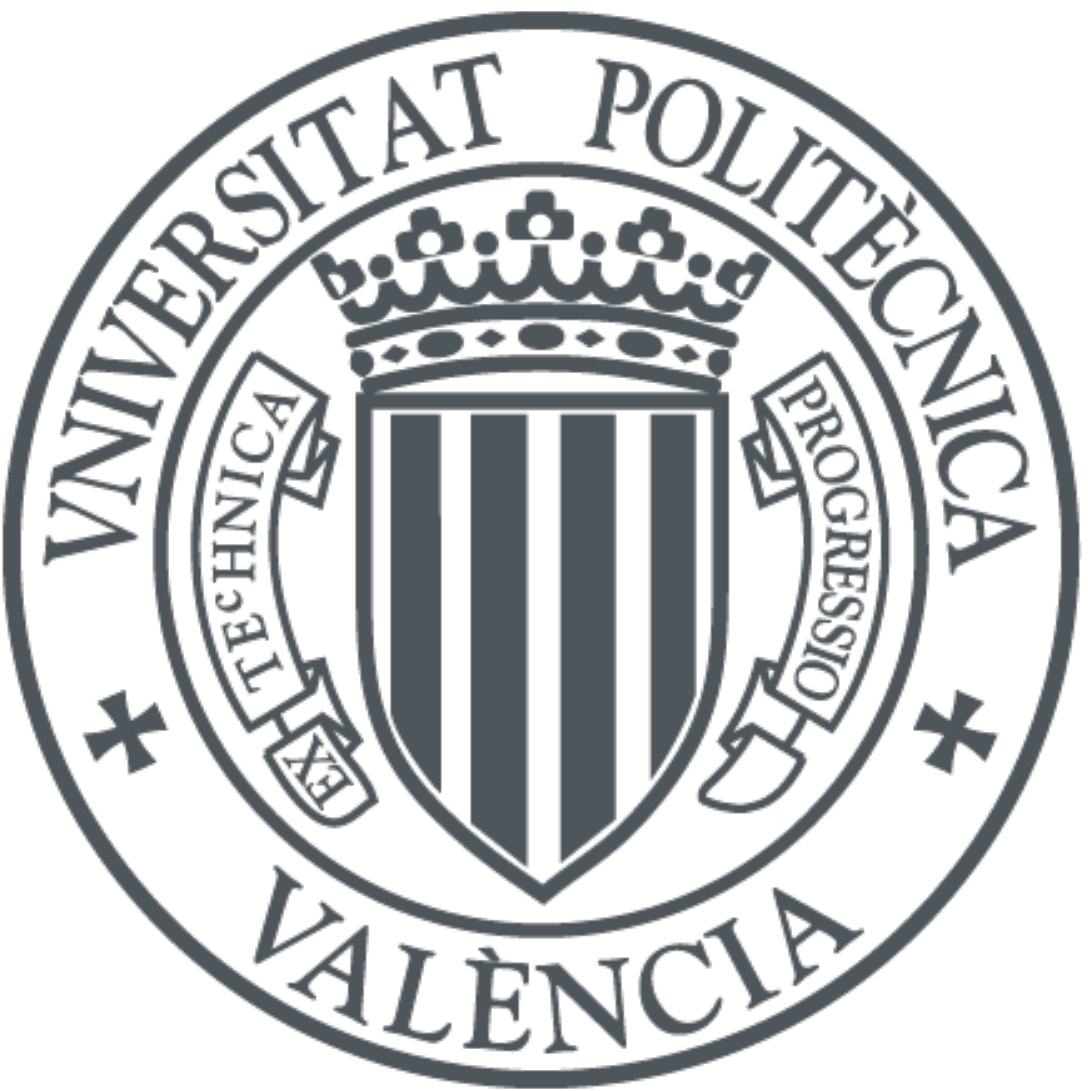

The final publication is available at

https://doi.org/10.1364/CLEO_SI.2020.STu3R.1

Copyright IEEE

Additional Information 


\title{
Space-Division Multiplexing Fibers for Microwave Signal Processing
}

\author{
Mario Ureña, Sergi García and Ivana Gasulla \\ ITEAM Research Institute, Universitat Politècnica de València, Camino de Vera s/n, 46022 Valencia, Spain \\ ivgames@iteam.upv.es
}

\begin{abstract}
We report the implementation of tunable microwave signal processing based on sampled true time delay line operation using a dispersion-engineered heterogeneous multicore fiber link. (c) 2020 The Author(s)
\end{abstract}

\section{Introduction}

Beyond high-capacity digital communications, multicore fibers (MCFs) [1] exhibit great potential in radio-overfiber distribution and microwave signal processing scenarios, [2]. In this context, we have previously proposed the use of dispersion-engineered heterogeneous MCFs as compact media for fiber-distributed signal processing, i.e., the simultaneous processing and distribution of microwave signals [3,4]. This approach is based in the custom design of MCFs to act as tunable sampled true time delay lines (TTDLs) where every core provides at the output a different time-delayed version of the radiofrequency signal. TTDLs are actually the basis of many microwave signal processing functionalities, such as signal filtering, radio beam-steering and optoelectronic oscillation [2]. For the MCF to operate as a TTDL, it must fulfil: (1) constant differential group delay between consecutive cores at a given wavelength; (2) constant differential chromatic dispersion between consecutive cores to enable tunability with the optical wavelength, and (3) low crosstalk, [4]. In this work, we report the design, fabrication and experimental demonstration of tunable sampled TTDL operation implemented on a 5-km heterogeneous MCF. The fiber comprises 7 different trench-assisted cores with different core dopant concentrations and dimensions. We experimentally demonstrate distributed microwave signal filtering and radio beam-steering in phased array antennas.

\section{Heterogeneous multicore fiber design and fabrication}

Following the above-mentioned requirements for group delay and chromatic dispersion, we designed a heterogeneous 7-core MCF to operate as a 7-sample true time delay line, [5,6]. The refractive index profile of each core consists of $\mathrm{a} \mathrm{GeO}_{2}$-doped core (with a different core radius $a_{1}$ and core-to-cladding relative index difference $\Delta_{1}$ ) surrounded by a silica inner cladding (with a different core-to-trench distance $a_{2}$ ) and a $1 \%$-Fluorine-doped trench (with a different width $w$ ). By tailoring all these design parameters in each core, we set a range of chromatic dispersion $D$ from 14.3 up to $20.3 \mathrm{ps} / \mathrm{km} / \mathrm{nm}$ (with a $1-\mathrm{ps} / \mathrm{km} / \mathrm{nm}$ incremental step), respectively for cores 1 up to 7 , and a common group delay to all cores at an anchor wavelength $\lambda_{0}=1530 \mathrm{~nm}$. The cladding-to-trench relative index difference is $0.321 \%$, the core pitch is $40 \mu \mathrm{m}$ and the cladding diameter is $150 \mu \mathrm{m}$.

The company YOFC fabricated a 5-km link of this heterogeneous MCF by using 7 different preforms. Fig. 1 (a) shows the photograph of the cross-sectional area of the MCF. Fig. 1 (b) shows the spectral differential group delays between core 7 and the rest, $\tau_{7}-\tau_{n}$, where markers correspond to the experimental values and lines to the theoretical ones. We see the measured differential group delays of cores 3 to 7 match the designed values, while there are some discrepancies for cores 1 and 2, which are the smallest ones and thus most sensitive to fabrication errors. The measured worst-case crosstalk for the 5-km MCF link is below $-30 \mathrm{~dB}$ while the average insertion losses are $5.8 \mathrm{~dB}$.

a)

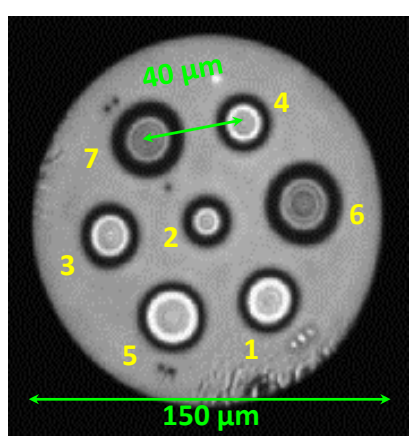

b)

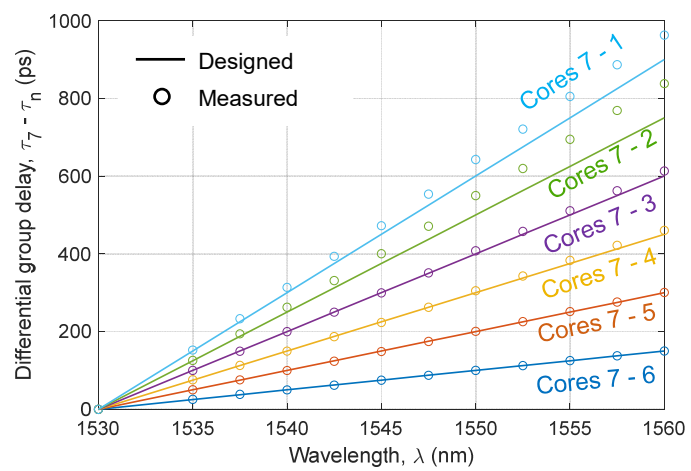

Fig. 1. a) Photograph of the fabricated MCF. b) Measured (markers) and computed (lines) spectral differential group delay for all the cores. 


\section{Application to microwave signal filtering and optical beamforming for phased array antennas}

First, we experimentally demonstrated the performance of the TTDL when it is applied to microwave signal filtering. Fig. 2 (a) shows the experimental setup used to measure the RF filtering response When we exploit the fiber space diversity, a single optical carrier is modulated and injected in all the cores, so that the different path delays come from the different fiber cores. For the wavelength dimension, an array of lasers operating at different wavelengths are modulated and injected to one of the cores, so that the different path delays correspond to the different wavelengths. Fig. 2 (b) shows, for instance, the measured frequency response for the 5-tap filter implemented with cores 3 to 7 (i.e. space diversity) for different operation wavelengths. We see the filter can be reconfigured since the free spectral range changes when tuning the optical wavelength.

Secondly, we demonstrated radio beam-steering in phased array antennas using the setup depicted in Fig. 3(a) to measure the phase difference between the photodetected RF signals provided by the different cores. Fig. 3 (b) shows the array factor we computed of a 5-element antenna (using cores 3 to 7) for an RF frequency of $10 \mathrm{GHz}$ and different optical wavelengths, where we can appreciate beam-pointing angle tunability.

a)

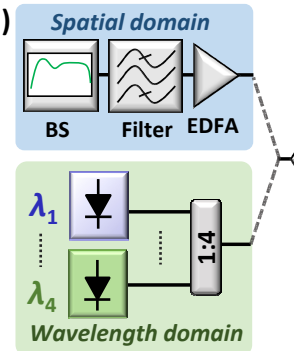

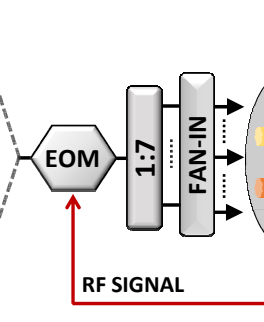

7-core MCF

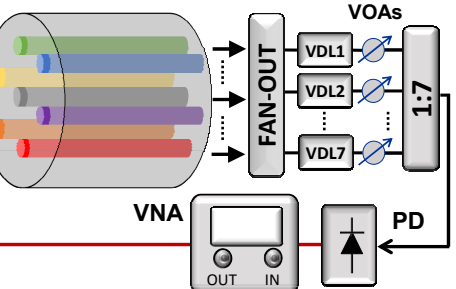

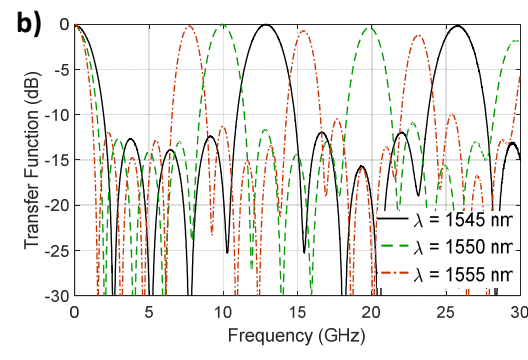

Fig. 2. a) Experimental setup for the RF filter. b) Measured RF filtering responses operating in spatial diversity for different wavelengths.

a)

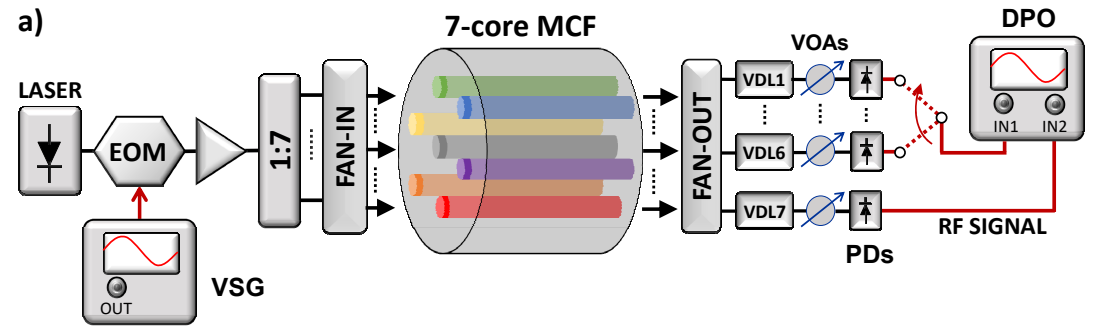

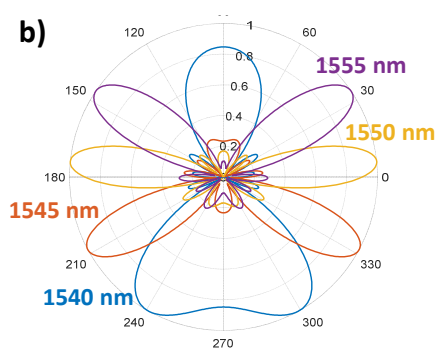

Fig. 3. a) Experimental setup for optical beamforming in phased array antennas. b) Simulated array factor AF with the measured true time delay values for an RF frequency of $10 \mathrm{GHz}$ and different optical wavelengths.

\section{Conclusions}

We showed here the design, fabrication and experimental demonstration of fiber-distributed microwave signal processing over a heterogeneous multicore fiber link. In particular, reconfigurable microwave signal filtering and optical beamforming for phased array antennas are demonstrated where a 5-km MCF act as the tunable true time delay line element. The MCF comprises 7 different trench-assisted cores that are different in terms of core dopant concentration as well as core and trench dimensions. This approach can be extended to perform additional microwave or optical signal processing applications that are based on time-discrete approaches.

\section{Acknowledgements}

This research was supported by the ERC Consolidator Grant 724663, Spanish MINECO Project TEC2016-80150-R, Generalitat Valenciana (PROMETEO 2017/017 research excellency award); Spanish MINECO scholarship BES2015-073359 for S. García and Spanish MINECO fellowship RYC-2014-16247 for I. Gasulla.

[1] D. J. Richardson, J. M. Fini and L. E. Nelson, “Space-division multiplexing in optical fibres,” Nat. Photonics 7, 354-362 (2013).

[2] J. Capmany et al., "Microwave photonic signal processing," IEEE J. Lightw. Technol. 31(4), 571-586 (2013).

[3] I. Gasulla and J. Capmany, "Microwave photonics applications of multicore fibers," Photonics J. 4(3), 877-888 (2012).

[4] S. García and I. Gasulla, "Dispersion-engineered multicore fibers for distributed radiofrequency signal processing," Opt. Express 24, 2064120654 (2016)

[5] S. García, M. Ureña and I. Gasulla, "Demonstration of distributed radiofrequency signal processing on heterogeneous multicore fibres", 45th European Conference on Optical Communication (ECOC 2019), Dublin, Ireland, 2019.

[6] S. García, M. Ureña and I. Gasulla, "Heterogeneous multicore fiber for optical beamforming", 2019 International Topical Meeting on Microwave Photonics (MWP2019), Ottawa, Canada, 2019. 